\title{
COMPARATIVO DE RENDIMIENTO DE VARIEDADES EXPERIMENTALES DE CAUPI
}

(*) CARDAMA VASQUEZI.

\section{RESUMEN}

El caupí (Vigna unguiculata w.), representa el mayor volumen de producción $(60 \%)$ en el departamento de Loreto, comparado con otras leguminosas de grano. Su cultivo se realiza con variedades tradicionales de características agronómicas poco deseables. Estas consideraciones motivaron que se ensayara variedades experimentales y locales, tendientes a obtener cultivares de alta capacidad productiva.

Los experimentos se ejecutaron en el campo experimental Muyuy (suelos inundables), perteneciente a la estación experimental agraria "San Roque" Iquitos, por espacio de cuatro años, comprendidos entre 1980 y 1983.

Del estudio se desprende la buena performance de las líneas experimentadas, las mismas que fueron comparadas teniendo en cuenta el rendimiento promedio con respecto al testigo local, sobresaliendo la Molina 1 con 1,787 Kg/ha., seguida de Chiclayo negro con $1,456 \mathrm{Kg} / \mathrm{h}$. las que, además, presentaron hábito de crecimiento semi-erecto y precocidad, así como tolerancia a plagas y enfermedades; de estas variedades experimentales sobresalientes, la M olina 1 mostró el grano marrón, color deseado por al productor y consumidor de la región, mientras que el Chiclayo negro presentó grano negro, por lo que se recomienda difundir el cultivo de la Molina 1, teniendo como al ternativa el Chiclayo negro.

\section{SUM M ARY}

The cowpea represents the mejor part $(60 \%)$ of the volume of production in the Loreto department compared with other leguminons grains. It is cultivated through the use of traditional types of agronomy whose characteristics are indesireable. This considerations caused local and experimental varieties to be tried in order to obtain methods of cultivation with a high yeld.

Ingo A grónomo, investigador agrario en el cultivo de leguminosas de granos, EEA "San Roque" Iquitos. Trabajo realizado convenio IIA P-CIPA XXII Loreto. 
The experiment was performed in the experimental area M uyuy (flooded land) which belongs to agraria experimental station "San Roque" Iquitos for 4 years between 1980 anual 1983.

The results have shown a betler performance improved varietes as compared witb the local ones. On the top was la M olina 1 variety whith $1787 \mathrm{Kg} / \mathrm{Ha}$. followed by Chiclayo negro with $1496 \mathrm{Kg}$./Ha. These two varietes are precocions and semi $4 \sim$ rected in growth habit. Grain color was brown in M olina 1 and black in Chiclayo negro. La molina 1 is recommen ded for distribution among farmer keeping Chiclayo negro as a second alternative.

Palabras claves: V ariedades experimentales, producción caupí.

\section{INTRODUCCION}

El caupí (Vigna unguiculata W.), fríjol común (Phaseolus vulgaris L.) y maní (Arachis hypogea $L$ ) son las leguminosas de grano de mayor arraigo popular entre los consumidores de la región; dentro de éstas, el caupí representa aproximadamente el $60 \%$ del volumen total de producción del departamento de Loreto (5), lo cual evidencia la gran importancia que representa para el poblador, principalmente rural de la selva por constituir una fuente económica de proteína fácil adquisición para las clases populares.

Actualmente, el productor de caupí en Loreto, utiliza para la siembra, entre otras, variedades criollas como garbanzo, pindayo, libaño y ucayalino, con características agronómicas poco deseables, tales como hábito ramador y periodo vegetativo tardío, siendo susceptible a enfermedades, principalmente virosis (virus del mosaico rugoso del caupí), pudriciones radiculares (M acrophomina sp, Rizoctonia sp, Phytium sp, etc.) y mustia hilachosa (Thanatephorus cucumeris), lo que ocasiona producciones relativamente bajas, estando alrededor de los $800 \mathrm{Kg} / \mathrm{ha}$. en promedio (7).

Esta situación permite evidenciar que el éxito de una agricultura progresista radica en la utilización de variedades mejoradas, que garanticen la resistencia 0 tolerancia a factores limitativos de producción, así como el potencial productivo que representa; al respecto Robles (4) manifiesta que, para asegurar una buena cosecha, se debe usar semilla certificada de variedades mejoradas, las cuales rinden mejor que las criollas, resisten las enfermedades, maduran uniformemente, tienen semillas del mismo color y son bien aceptadas en el mercado. 
Como antecedentes de trabajo similares en Iquitos, se puede mencionar que M endoza (3) en 1978, al realizar un comparativo de 15 variedades de caupí, encontró que una de las mejores variedades era la "M olina 1" con un rendimiento promedio de 1,986 Kg/ha., seguida de EE.UU. - 1- $211(1,558 \mathrm{Kg} / \mathrm{H}$ a) y EE.UU.- 1 - 208 (1,448 $\mathrm{Kg} / \mathrm{ha})$, superando al tipo local garbancillo $(664 \mathrm{Kg} / \mathrm{ha})$; mientras que en el siguiente año, sobresalieron EE.UU . - 1 - 216, colorado y cáscara negra, con 1,936, 1,833 y $1,726 \mathrm{Kg} / \mathrm{ha}$., respectivamente.

Zumaeta (6) en un ensayo de 10 variedades de caupí en Iquitos, reporta como las mejores en rendimientos de grano seco a la Filipinas $1-3(1,822 \mathrm{Kg} / \mathrm{Ha})$ y Porvenir $1(1,670 \mathrm{Kg} / \mathrm{ha})$, las que, además, mostraron precocidad y crecimiento vegetativo determinado.

Ferreyra, de A sia Feliciano y Da Silva (2); comparando cultivares de caupí de diversos hábitos de crecimiento en el territorio federal de A mapá, determinaron que el mejor rendimiento lo alcanzó el genotipo introducido IPEANV-69, con 997 $\mathrm{Kg} / \mathrm{ha}$., superando en $77 \%$ al cultivar V -2 CR Vagen branca ( $897 \mathrm{Kg} / \mathrm{ha}$ ), seguido de garoto $(719 \mathrm{Kg} / \mathrm{ha})$.

Estas razones indujeron a conducir experimentaciones en caupí, con variedades locales e introducidas, con la finalidad de obtener cultivares de alta capacidad productiva.

\section{MATERIALES Y METODOS}

Cuatro (4) estudios comparativos, de variedades de caupí de diferentes hábitos vegetativos, fueron conducidos en el campo experimental M uyuy, dentro del Período 1980 a 1983, en suelos aluviales de restinga baja ( inundables periódicamente), de textura franco arcillo limoso, caracterizado por su acidez (5.32), materia orgánica baja (1,03\%), fósforo medio (7.8 ppm) y potasio bajo (55.25 ppm.)

Se utilizó el diseño experimental de tipo block completo randomizado con cuatro repeticiones y quince tratamiento hasta 1981, pasando a 3 repeticiones y dieciséis tratamientos hasta 1983. Las parcelas tuvieron cuatro hi eras de $6.0 \mathrm{~m}$, de I'.rgo, separadas $0.60 \mathrm{~m}$. entre cada una de ellas y distanciadas $0.40 \mathrm{~m}$. entre plantas, con una densidad poblacional de 3 plantas por mata.

Hubo incidencia de plaga', principalmente de gusanos de tierra (Agrotis sp. y Feltia sp.) y grillos, en inicio de crecimiento de las plantas, los que fueron controladas con Aldrín $2.5 \%$, en forma de espolvoreo; durante todo el período vegetativo 
del cultivo se presentaron crisomélidos, cuyo control efectivo' se hizo con Sevín 85\% P.M. al $0.3 \%$.

Como enfermedades se presentaron pudriciones radiculares (Rhizoctonia Phytium), manchas foliares (Alternaria y Cercóspora) y virosis, causada por el virus del mosaico rugoso del caupí, que fue el de mayor importancia. Los potenciales de producción de los cultivares evaluados fueron comparados con la variedad local garbanzo en cada año, determinándose el coeficiente de la variación para cada estudio mediante la fórmula siguiente:

A nalizando los rendimientos a través de las prueba de Tuckey (5\%), se observa que la Molina 1 fue superior a la variedad local garbanzo, así como a cáscara negra, dosmesinos, colorado y Filipinas 1-3.

\section{RESULTADOS}

Se analizaron en forma individual y combinada los experimentos, a través de las producciones medias, expresadas en $\mathrm{Kg} / \mathrm{Ha}$., asimismo, se establecieron las características agronómicas más importantes de los materiales genéticos evaluados, que tuvieron continuidad durante los 4 años de experimentación.

Como complemento a la información, se añadió los índices de rendimientos, considerando a la variedad local garbanzo como el $100 \%$, para observar el adelanto del resto de cultivares que, en su mayoría, fueron introducidos.

El Cuadro 1 muestra los resultados del experimento conducido en 1980, sobresaliendo el cultivo la Molina 1 sobre el resto; tuvo el menor rendimiento la variedad de Filipinas 1-3, incluso por debajo del testigo local garbanzo.

$$
\mathrm{CV}=\frac{V_{\mathrm{CMe}}}{\mathrm{X}} \times 100
$$




\section{CUADRO 1. RESULTADOS OBTENIDOS DEL ESTUDIO CONDUCIDO EN 1980}

\begin{tabular}{llll}
\hline Cultivar & $\begin{array}{l}\text { Rendimiento } \\
\text { Grano seco } \\
\text { Kg./ha. }\end{array}$ & $\begin{array}{l}\text { Significancia } \\
\text { (Tuckey 5\%) }\end{array}$ & $\begin{array}{c}\text { I } \\
(\%)\end{array}$ \\
\hline & & & \\
La M olina 1 & 29088 & a & 217 \\
Porvenir 1 & 1.656 & ab & 172 \\
EE.UU. -I-208 & 1,643 & a b & 171 \\
Chiclayo Negro & 1,525 & a b c & 158 \\
EE, UU. -I-217 & 1,483 & a b c & 154 \\
EE.UU. -I-21I & 1,456 & a b c & 151 \\
EE.UU. -I-275 & 1,427 & a b c & 148 \\
EE.UU. -I-241 & 1,305 & a b c & 136 \\
Local-1 & 1,286 & a b c & 134 \\
EE.UU. -I-219 & 1,246 & a b c & 129 \\
Garbanzo & 962 & b c & 100 \\
Cáscara negra & 874 & b c & 91 \\
Dosmesinos & 802 & b c & 83 \\
Clorado & 758 & c & 79 \\
Filipinas 1-3 & 533 & c & 55 \\
& & & \\
\hline
\end{tabular}

$\begin{array}{ll}C . V . & =28 \% \\ X & =1,270 \mathrm{Kg} / \mathrm{h} .\end{array}$

Producciones con letras iguales son semejantes estadísticamente.

En el segundo experimento (Cuadro 2), instalado en 1981, también la M olina 1 fue el genotipo que logró la mayor producción de grano, pero sin significancia estadística con los demás genotipos puestos a comparación; en este año, cáscara negra fue el de menor rendimiento 
80

CUADRO 3 RENDIMIENTOS OBTENIDOS DEL ESTUDIO CONDUCIDO EN 1982

\begin{tabular}{llll}
\hline Cultivar & $\begin{array}{l}\text { Rendimiento } \\
\text { Grano seco } \\
\text { Kg./ha. }\end{array}$ & $\begin{array}{l}\text { Significancia } \\
\text { (Tuckey 5\%) }\end{array}$ & $\begin{array}{c}\text { । } \\
(\%)\end{array}$ \\
\hline & & & \\
La M olina 1 & 1,662 & a & 292 \\
Chiclayo negro & 1,548 & a & 272 \\
Seda & 1,362 & a & 239 \\
EE.UU-I-211 & 1.351 & a & 237 \\
Porvenir. 1 & 1,250 & a b & 219 \\
Local 1 & 1,216 & a b & 213 \\
EE.UU.-1-219 & 1,121 & a b & 197 \\
Filipinas-3 & 1,082 & a b & 190 \\
R-8-81 & 1,039 & a b & 182 \\
EE.UU.-I-275 & 915 & a b & 122 \\
EE.UU.-I--241 & 859 & a b & 151 \\
Dosmesinos & 674 & a b & 118 \\
EE.UU.-I-216 & 662 & a b & 116 \\
Garbanzo & 570 & a b & 100 \\
Colorado & 179 & b & 31 \\
EE.UU.-I-208* & $-\cdots$ & $-\cdots$ & --- \\
& & & \\
\hline
\end{tabular}

* Perdido por escaso poder germinativo

C.V. $=36 \%$

$\mathrm{X}=1,033 \mathrm{Kg} . / \mathrm{ha}$.

Producciones con letras iguales no difieren estadísticamente

En el cuadro 4, son presentados los rendimiento obtenidos en el cuarto experimento, que corresponde al año 1983; en ésta ocasi6n, destacó por su mayor producción el cultivar seda, seguido Chiclayo negro y la M olina 1, quienes mostraron superioridad estadística al testigo local garbanzo, así como a L-4-81, Porvenir 1, y EEUU -I-208, los mismos que lograron los menores rendimientos. 


\section{CUADRO 4 RENDIMIENTOS OBTENIDOS DEL ESTUDIO CONDUCIDO EN 1983}

\begin{tabular}{|c|c|c|c|c|}
\hline Cultivar & $\begin{array}{l}\text { Rendimiento } \\
\text { Grano- seco } \\
\text { Kg./ha. }\end{array}$ & $\begin{array}{l}\text { Significancia } \\
\text { (Tuckey }\end{array}$ & $\begin{array}{c}1 \\
5 \%) \\
\end{array}$ & $(\%)$ \\
\hline Seda & 1,385 & $a$ & 255 & \\
\hline Chiclayo negro & 1,290 & $a$ & 238 & \\
\hline La M olina 1 & 1,277 & $a$ & 235 & \\
\hline EE.UU.-I-275 & 1,235 & $a b$ & 227 & \\
\hline EE.UU.-I-216 & 1,213 & $a b$ & 223 & \\
\hline EE.UU.-I-241 & 1,193 & $a b c$ & 220 & \\
\hline Local - 1 & 1,191 & $a b c$ & 219 & \\
\hline Garbancillo pardo & 1,034 & $a b c$ & 190 & \\
\hline$R-8-81$ & 900 & $a b c$ & 166 & \\
\hline Filipinas -3 & 816 & $a b c$ & 150 & \\
\hline Garbanzo jaspeado & 766 & $a b c$ & 141 & \\
\hline TVX-1,952 DIE (Africano) & 546 & $a b c$ & 101 & \\
\hline Garbanzo (Testigo local) & 543 & bc & 100 & \\
\hline$L-4-81$ & 521 & bc & 96 & \\
\hline Porvenir 1 & 432 & c & 80 & \\
\hline EE.UU.-I-208 & 345 & c & 64 & \\
\hline
\end{tabular}

$\begin{array}{ll}\text { C.V. } & =26 \% \\ X & =918 \mathrm{Kg} . / \mathrm{ha} .\end{array}$

Producciones con letras iguales son semejantes estadísticamente.

En los Cuadros 5 y 6 . se presentan los resultados de los cultivares que secuencialmente fueron probados durante los cuatro años de experimentación, sobresaliendo por su rendimiento la Molina 1, superando en más de $900 \mathrm{Kg} / \mathrm{ha}$. a la variedad local garbanzo. 
CUADRO 5 RENDIMIENTOS OBTENIDOS DE LOS CULTIVARES EVALUADOS QUE TUVIERON CONTINUIDAD LOS AÑOS DE EXPERIMENTACION

Orden
de Cultivares

Mérito

$\begin{array}{llll}1980 & 1981 & 1982 & 1983\end{array}$

Total Promedio ।

\begin{tabular}{llrrrrrrr}
\hline 01 & La Molina 1 & 2,088 & 2,121 & 1,662 & 1,277 & 5,871 & 1,787 & 215 \\
02 & Chiclayo negro & 1,525 & 1,426 & 1,584 & 1,291 & 5,025 & 1,456 & 175 \\
03 & Local 1 & 1,286 & 1,807 & 1,216 & 1,191 & 5,500 & 1,375 & 165 \\
04 & EE.UU. I-275 & 1,427 & 1,854 & 915 & 1,235 & 5,431 & 1,358 & 163 \\
05 & EE.UU. I-216 & 1,525 & 2,024 & 662 & 1,213 & 5,424 & 1,356 & 163 \\
06 & Porvenir 1 & 1,656 & 1,884 & 1,250 & 432 & $5,22.2$ & 1,306 & 157 \\
07 & EE.UU I-241 & 1,305 & 1,637 & 859 & 1,193 & 4,994 & 1,249 & 150 \\
08 & Filipinas -3 & 533 & 1,966 & 1,082 & 816 & 4,397 & 1,099 & 132 \\
09 & Garbanzo (TL) & 962 & 1,250 & 570 & 543 & 3,325 & 831 & 100 \\
10 & EE.UU. I-208 & 1,643 & 1,247 & $(\%)$ & 334 & 3,235 & 809 & 97 \\
& & & & & & & & \\
\hline
\end{tabular}

(*) Perdido por escaso poder germinativo 
CUADRO 6 PRINCIPALES OBSERVACIONES REGISTRADAS A LOS CULTIVARES EVALUADOS SECUENCIALMENTE DURANTE LOS CUATRO AÑOS DE EXPERIMENTACION

\begin{tabular}{llllllc} 
Cultivares & $\begin{array}{l}\text { Hábito } \\
\text { Vegeta- } \\
\text { tivo }\end{array}$ & $\begin{array}{l}\text { Período } \\
\text { Vegetativo } \\
\text { (días) }\end{array}$ & $\begin{array}{l}\text { Plagas y } \\
\text { enferme- } \\
\text { dades }\end{array}$ & $\begin{array}{c}\text { Grano } \\
\text { Vaina } \\
\text { (Promedio) }\end{array}$ & $\begin{array}{l}\text { Peso } \\
\text { semilla } \\
\text { (gr.) }\end{array}$ & $\begin{array}{l}\text { Color de } \\
\text { grano }\end{array}$ \\
\hline Porvenir 1 & S. E. & 80 & Tolerante & 14 & 14 & M arrón claro \\
Local 1 & S. R. & 85 & Susceptible & 14 & 12 & M arrón \\
Filipinas-3 & S. E. & 76 & Tolerante & 15 & 14 & Marrón \\
La Molina 1 & S. E. & 82 & Tolerante & 16 & 15 & Marrón \\
EE.UU I-275 & S. R. & 88 & Tolerante & 17 & 14 & Marrón \\
Garbanzo & R. & 91 & Susceptible & 17 & 16 & Pardo \\
EE.UU I-208 & S.E & 83 & Tolerante & 15 & 13 & Blanco \\
Chiclayo negro & S. E. & 83 & Tolerante & 16 & 14 & N egro \\
EE.UU.I-241 & S. R. & 73 & Susceptible & 16 & 20 & Marrón \\
EE.UU.I-216 & S. R. & 87 & Tolerante & 19 & 12 & Rojizo
\end{tabular}

Semi rastrero $=S . R$.

Semi erecto $=\mathrm{S}$. $\mathrm{E}$.

Rastrero $=\mathrm{R}$. 


\section{DISCUSION}

L os experimentos fueron conducidos en diferentes años y tuvieron lugar en una misma localidad, bajo condiciones de clima y suelo similar; sin embargo, a pesar del escaso número de experimentos realizados por cada año, el desenvolvimiento comparativo de los cultivares en relación al Testigo local garbanzo, mayormente fue superior, tal como se puede apreciar en los Cuadros 1 al 5, así como en el Cuadro 6, que reporta las principales observaciones registradas a los cultivares que tuvieron continuidad en el transcurso de los años sometidos a estudio. En el primer año de experimentación (1980), el cultivar la Molina 1 destacó nítidamente por su rendimiento, superando con el doble de la producción a la variedad local garbanzo, el cual fue utilizado como testigo por su mayor difusión en la zona; asimismo, Porvenir1, EE.UU-I-208 y Chiclayo negro reportaron rendimientos espectantes, perfilándose como cultivares promisorios.

En 1981, las producciones de los materiales genéticos evaluados fueron superiores al de la campaña anterior, debido a la mejor viabilidad y vigor que presentaron las semillas al momento de la germinación, así como a los menores problemas fitosanitarios presentados durante la conducción del experimento. En este año, nuevamente la Molina 1 tuvo un comportamiento superior en producción de granos, respecto a la variedad local garbanzo, incluyendo a los demás materiales genéticos; sin embargo, se aprecia que EE.UU-I-216, Filipinas 1- 3, Porvenir- 1, EE.UU-I-275, y local 1, tuvieron desenvolvimiento productivamente semejantes a la Molina 1. En el experimento del año de 1982, la Molina 1 reeditó su performance productiva, seguido con rendimientos similares de Chiclayo negro, Seda, EE.UUI-211, perdiéndose al momento de la siembra EE.UU-I-208, por bajo poder germinativo. Un detalle que se puede notar en este experimento, es el relativamente elevado coeficiente de variabilidad presentado, debiéndose mas que todo a factores genéticos que a factores ambientales, por los diversos materiales genéticos estudiados con diferentes características agronómicas; éste mis- ¡no factor de la variabilidad intergene -típica Da Ponte (1) en Belém-Brasil, ya se había determinado al comparar 16 cultivares del tipo erecto, con un rango de producción de 927 a 1,381 K g/ha. En el último año de experimentación (1983), obtuvo la mayor producción el cultivar Seda, seguido de Chiclayo negro, la Molina 1 y EE.UU-I-275, cuyos rendimientos fueron significativos comparativamente con la del garbanzo (testigo local). En esta ocasión, la Molina 1 no tuvo la mayor producción, pero, a pesar de ello, su rendimiento mantiene similitud con los cultivares que le superaron.

El Cuadro 5, registra un resumen de los rendimientos de todos los materiales genéticos que tuvieron continuidad en su evaluación durante los 4 años de 
comparación, destacando la M olina 1, seguido de Chiclayo negro, con rendimientos superiores a las demás variedades experimentales, incluyendo a la variedad local garbanzo.

De acuerdo a las observaciones registradas (Cuadro 6), se aprecia que la Molina 1 y Chiclayo negro, variedades que lograron los mayores rendimientos, muestran hábito vegetativo semi-erecto, precocidad y tolerancia a plagas y enfermedades, características agronómicas consideradas como deseables, dentro de los objetivos de investigación en suelos aluviales de restinga baja en el Programa de L eguminosas de Grano.

El productor y consumidor de caupí de la región, prefiere mayormente granos de colores marrón, pardo, crema o blanco; en este sentido, la M olina 1, por presentar los granos de color marrón, estaría encuadrado dentro de la preferencia del poblador amazónico, mientras que Chiclayo negro, por presentar el grano con la testa negra, podría ser considerado como una alternativa mas para el consumo regional, así como para exportación a otros lugares del país que carecen de menestras y también a nivel internacional, donde el grano de color negro tiene buena aceptación.

\section{CONCLUSIONES}

Después de 4 años de experimentación y analizando comparativamente el rendimiento de grano seco de los materiales genéticos evaluados, se pueden plantear las siguientes conclusiones:

La M olina 1, por presentar buen rendimiento de grano seco, resulta una de las mejores alternativas dentro de la región para desarrollar su cultivo. Chiclayo negro es otra de las variedades experimentales que reportó buen rendimiento después de la Molina 1.

Garbanzo, testigo local, fue superado en rendimiento en 75 y $115 \%$ por las variedades experimentales Chiclayo negro y la M olina 1 , respectivamente, poniendo de manifiesto que existen cultivares mejorados de mayor potencial productivo que las criollas. Las variedades experimentales la Molina 1 y Chiclayo negro, que sobresalieron por su rendimiento, presentan buenas características, tales como hábito vegetativo semi-erecto y precocidad, así como tolerancia a plagas y enfermedades. 